WE3F-G4

\title{
AN IMPROVED 2D-FDTD METHOD FOR TWFET BANDWIDTH CHARACTERIZATION
}

\author{
Carlo Angiolini, Guglielmo D'Inzeo, Pasquale Rota
}

\author{
Electronic Engineering Department - "La Sapienza” University of Rome, Italy
}

\begin{abstract}
A new 2D-FDTD method has been proposed to analyze the dispersion diagram of planar circuits. Traveling wave field effect transistor (TWFET) is a solid state device designed to amplify signals over a wide bandwidth. An analysis of the passive behavior of this device has been performed using mode-matching technique and assuming that the passive structure performances are affected only by the width of the $\mathrm{T}$ bar of the gate electrode. To verify such hypothesis and to determine the cut-off frequency of the higher order modes that limits its bandwidth, we have simplified the 2D-FDTD method with a particular normalization, that permits the analysis of the case $\beta=0$.

The proposed approach was tested calculating the dispersion for some known structures and it has been used in the TWFET characterization
\end{abstract}

\section{INTRODUCTION}

Traveling wave field effect transistor (TWFET) (Fig. 1) is a solid state device designed to amplify signals over a wide bandwidth. It can be considered as a distributed MESFET, where the amplification effect of field effect transistor is used in a distributed interaction with the electromagnetic field, typical of the traveling wave tubes.

An analytical model of this structure has been introduced in [1], where it is also shown that, to obtain amplification, it is necessary to have a T-shape crosssection of the gate, permitting a higher transconductance and at the same time, a reduction of losses.

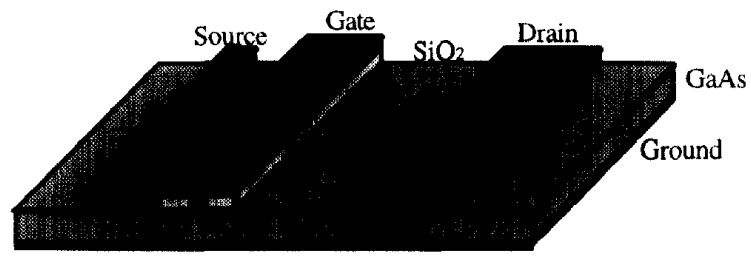

Fig. 1 Tridimensional global view of the TWFET
An analysis of the passive behavior of this device has been made in [2], using a perturbational approach modified to determine the losses. Such approach realized through the mode matching method and the transverse resonance technique studies the equivalent planar structure, assuming that the passive structure performances are affected only by the width of the $\mathrm{T}$ bar, and not by the height or width of the conductor below.

To verify such hypothesis and to determine the cutoff frequency of the higher order modes that limits its bandwidth, we have chosen to study the TWFET with a 2D-FDTD method $[3,4]$. This technique permits to obtain the dispersion curves of uniform guiding structures, by using a two-dimensional mesh in which the field components are set on the unit cell as shown in Fig. 2.

Since the analysis is made choosing a propagation constant $B$ and determining the corresponding frequencies, it is particularly useful to obtain the cut-off frequencies of the higher order modes.

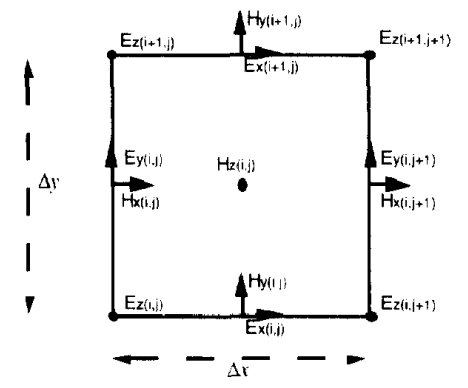

Fig.2 The unit cell of the compact 2D-FDTD

\section{THEORY}

In [5] it is shown that if in the 3D-FTDT we introduce a phase shift along the $z$-direction (propagation direction) of the phasors of the field components, we can represent the electromagnetic field in a two dimensional structure through a real coefficients equation system. The expressions of $\mathrm{H}_{\mathrm{x}}$ and $\mathrm{H}_{\mathrm{y}}$ are: 


$$
\begin{aligned}
& H_{y}{ }^{n+1 / 2}(i, j)=H_{y}{ }^{n-1 / 2}(i, j) \\
& +\frac{\Delta t}{\mu(i, j) \Delta x}\left(\beta \Delta x E_{x}^{n}(i, j)+E_{z}^{n}(i, j+1)-E_{z}^{n}(i, j)\right) \\
& H_{z}^{n+1 / 2(i, j)}=H_{z}^{n-1 / 2(i, j)} \\
& +\frac{\Delta t}{\mu(i, j) \Delta y}\left(E_{x}^{n}(i+1, j)-E_{x}^{n}(i, j)\right) \\
& -\frac{\Delta t}{\mu(i, j) \Delta x}\left(E_{y}^{n}(i, j+1)-E_{y}{ }^{n}(i, j)\right)
\end{aligned}
$$

that permits to consider only the real part of the field components.

In [6] it has been obtained the stability condition for the algorithm. In [7] a simple method to use a graded lattice has been introduced. Considering for example the case $\Delta y_{2}=3 \Delta y_{1}$ shown in Fig. 3 , it has been pointed out how the calculation of $E_{x}\left(i_{0}, j\right)$ and $E_{z}\left(i_{0}, j\right)$ can be obtained considering these components as belonging to the coarse mesh with a second order accuracy.

Starting from considerations exposed in [8], in this work we simplify the method introducing a particular normalization of the field $\vec{E}$. Observing that system (1) can be simplified in the hypothesis of a medium having a constant magnetic permeability, we introduce the normalized components of the electric field:

$$
\begin{aligned}
& \frac{\Delta t}{\mu \Delta y} E_{x}=\hat{E}_{x}(2 a) ; \frac{\Delta t}{\mu \Delta x} E_{y}=\hat{E}_{y}(2 b) ; \frac{\Delta t}{\mu \Delta x} E_{z}=\hat{E}_{z}(2 c) \\
& \text { Replacing (2) in (1), we obtain: } \\
& H_{x}{ }^{n+1 / 2(i, j)}=H_{x}^{n-1 / 2(i, j)} \\
& -\frac{\Delta x}{\Delta y}\left(\hat{E}_{z}^{n}(i+1, j)-\hat{E}_{z}^{n}(i, j)+\beta \Delta y \hat{E}_{y}^{n}(i, j)\right) \\
& H_{y}{ }^{n+1 / 2}(i, j)=H_{y}{ }^{n-1 / 2(i, j)} \\
& +\beta \Delta y \hat{E}_{x}^{n}(i, j)+\hat{E}_{z}^{n}(i, j+1)-\hat{E}_{z}^{n}(i, j) \\
& H_{z}{ }^{n+1 / 2}(i, j)=H_{z}{ }^{n-1 / 2}(i, j) \\
& +\hat{E}_{x}{ }^{n}(i+1, j)-\hat{E}_{x}{ }^{n}(i, j)-\hat{E}_{y}^{n}(i, j+1)+\hat{E}_{y}{ }^{n}(i, j) \\
& \hat{E}_{x}^{n+1}(i, j)=\hat{E}_{x}{ }^{n}(i, j)+\frac{\Delta t^{2}}{\mu \varepsilon(i, j) \Delta y^{2}} \\
& \cdot\left(H_{z}{ }^{n+1 / 2}(i, j)-H_{z}{ }^{n+1 / 2}(i-1, j)-\beta \Delta y H_{y}{ }^{n+1 / 2}(i, j)\right) \\
& \hat{E}_{y}^{n+1}(i, j)=\hat{E}_{y}^{n}(i, j)+\frac{\Delta t^{2}}{\mu \varepsilon(i, j) \Delta x^{2}} \\
& \cdot\left(\beta \Delta x H_{x}{ }^{n+1 / 2}(i, j)-H_{z}{ }^{n+1 / 2}(i, j)+H_{z}{ }^{n+1 / 2(i, j-1)}\right)
\end{aligned}
$$

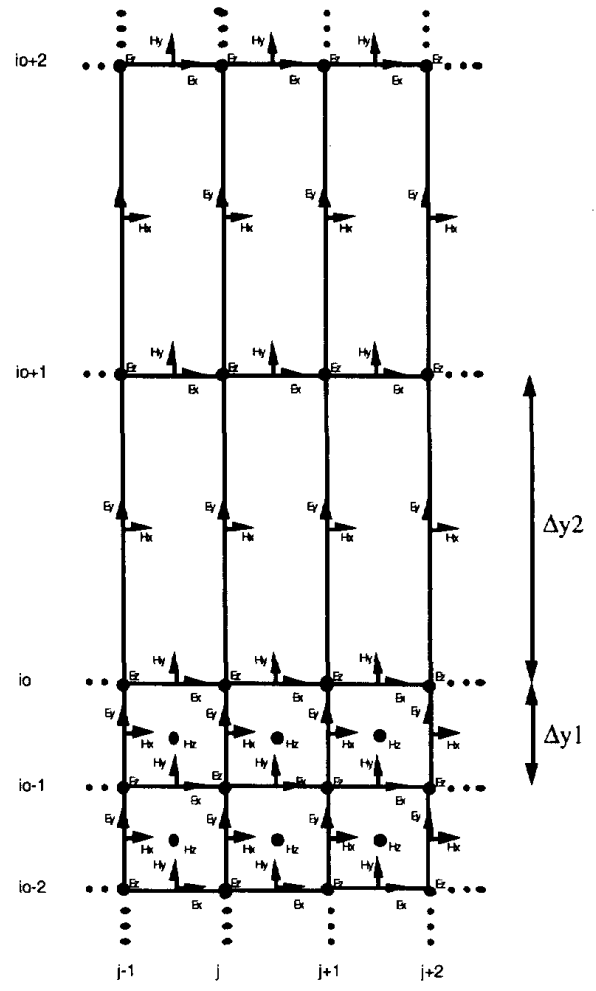

Fig.3 Graded lattice; $\Delta y_{2}=3 \Delta y_{1}$

$$
\begin{aligned}
& \hat{E}_{z}^{n+1}(i, j)=\hat{E}_{z}^{n}(i, j) \\
& +\frac{\Delta t^{2}}{\mu \varepsilon(i, j) \Delta x^{2}}\left(H_{y}{ }^{n+1 / 2}(i, j)-H_{y}{ }^{n+1 / 2}(i, j-1)\right) \\
& -\frac{\Delta t^{2}}{\mu \varepsilon(i, j) \Delta x \Delta y}\left(H_{x}{ }^{\left.n+1 / 2(i, j)-H_{x}{ }^{n+1 / 2}(i-1, j)\right)}\right.
\end{aligned}
$$

In such way we have reduced the number of computational operations per cell and per time step from 32 (20 sums plus 12 multiplications) to 29 (20 sums plus 9 multiplications), simply avoiding making superfluous calculations. The stability condition shown in [6] is still valid. With system (3) we determine the time behavior of the normalized components and not the absolute values of $\bar{E}$. Nevertheless it is not necessary to de-normalize these data, since to obtain the dispersion diagram we have just to determine the frequencies of the peaks in the Fourier spectrum of a field component in an arbitrary point of the structure.

Among all the available normalizations, the one we introduced permits the analysis of the case $\beta=0$. Moreover (2) have the advantage of an extra simplification when $\Delta x=\Delta y$. Thus from (3) we easily obtain an algorithm with 27 operations ( 20 sums plus 7 multiplications). Considering the particular case $\beta=0$, we obtain a system with just 21 operations ( 16 sums plus 5 multiplications), and, if $\Delta x=\Delta y, 19$ operations (16 sums plus 3 multiplications). 
In the graded lattice situation shown in fig. 3, we have to introduce two different normalizations for $E_{\mathrm{X}}$. In the fine mesh we pose:

$$
\frac{\Delta \mathrm{t}}{\mu \Delta \mathrm{y}_{1}} \cdot \mathrm{E}_{\mathrm{x}}=\hat{\mathrm{E}}_{\mathrm{xl}}
$$

while in the coarse mesh we have:

$$
\frac{\Delta t}{\mu \Delta y_{2}} E_{x}=\hat{E}_{x 2}
$$

Since they do not involve $\Delta y$, we can consider ( $2 b$ ) and $(2 \mathrm{c})$ too. System (3) is valid in each zone but boundary conditions treatment needs some more considerations.

We have already pointed out that what we have to calculate is $\mathrm{E}_{\mathrm{x} 2}\left(\mathrm{i}_{0}, \mathrm{j}\right)$. This component is present in the expression of $\mathrm{H}_{2}\left(\mathrm{i}_{0}, \mathrm{~J}\right)$ in the coarse mesh, and in those of $\mathrm{H}_{\mathrm{y}}\left(\mathrm{i}_{0}, \mathrm{j}\right)$ and $\mathrm{H}_{2}\left(\mathrm{i}_{0}-1, \mathrm{j}\right)$ in the fine mesh. The latter components need a proper normalization of $E_{x}\left(i_{0}, j\right)$

Replacing (4) in ( $3 b$ ) and ( $3 c)$ we obtain

$$
\begin{aligned}
& H_{y}{ }^{n+1 / 2}\left(i_{0}, j\right)=H_{y}{ }^{n-1 / 2}\left(i_{0}, j\right) \\
& +\beta \Delta y_{2} \hat{E}_{x 2}{ }^{n}\left(i_{0}, j\right)+\hat{E}_{z}{ }^{n}\left(i_{0}, j+1\right)-\hat{E}_{z}{ }^{n}\left(i_{0}, j\right) \\
& H_{z}{ }^{n+1 / 2}\left(i_{0}-1, j\right)=H_{z}{ }^{n-1 / 2}\left(i_{0}-1, j\right)+\hat{E}_{x 2}{ }^{n}\left(i_{0}, j\right) \frac{\Delta y_{2}}{\Delta y_{1}} \\
& -\hat{E}_{x 1}{ }^{n}\left(i_{0}-1, j\right)-\hat{E}_{y}{ }^{n}\left(i_{0}-1, j+1\right)+\hat{E}_{y}{ }^{n}\left(i_{0}-1, j\right)
\end{aligned}
$$

Equation (5a) needs as many operations as (3b), whereas $(5 \mathrm{~b})$ needs one more multiplication than $(3 \mathrm{c})$. Thus, apart the $\mathrm{H}_{2}\left(\mathrm{i}_{0}-1, \mathrm{j}\right)$ calculation, (4), (2b) and (2c) imply the same saving of computer time shown for the uniform mesh.

\section{RESULTS}

The proposed approach was tested calculating the dispersion of a NSSS (Non-symmetrical Suspended Substrate Stripline) studied using the transverse resonance method and experimentally verified in [9]. Our analysis was made using a $\Delta y$ variable step mesh of the kind described in fig. 3 , three times in the part above and once in the part below the dielectric substrate, reducing the number of cells from $200 \times 48$ to $66 \times 48$. The results shown in Fig. 4 are in excellent agreement both with experiments and with theoretical results from [9].

To further test the method we considered the study of shielded multilayer coplanar waveguides cut-off frequencies developed in [10] and [11].

The results in Fig. 5 show a good agreement for the fundamental mode to both the approaches [10] ad [11] Regarding the higher order mode, our results are closer to thos: reported in $[11]$. We tested such case with a commercial package HFSS (High Frequencies Structure Simulator) [12] that showed agreement with the results we obtained. As a consequence the results shown in [10] should be somehow incorrect.

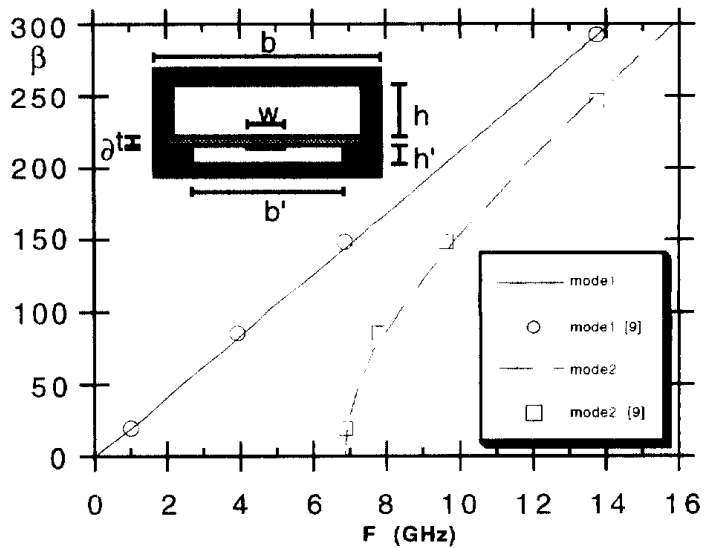

Fig.4 Dispersion diagram of the first two modes of a NSSS. Comparison with [9]. $\left(b=22.86 \mathrm{~mm}, b^{\prime}=15.8 \mathrm{~mm}\right.$. $\mathrm{h}=\mathrm{b} / 2, \mathrm{~h}^{\prime}=0.1 \mathrm{~b}, \varepsilon_{\mathrm{r}}=2.2, \mathrm{t}=0.254 \mathrm{~mm}, \delta=0.03 .5 \mathrm{~mm}$ )

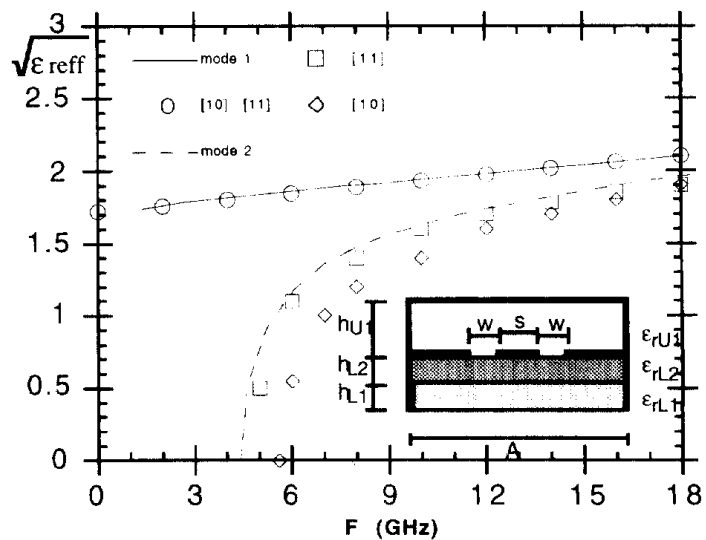

Fig.5 $\sqrt{\varepsilon_{\text {reff }}}$ of the dominant mode and first higher order mode as a function of frequency in a shielded multilayer CWG. Comparison with [10] and [11]. $\left(\mathrm{h}_{\mathrm{LI}}=\mathrm{h}_{\mathrm{U} 1}=4.5\right.$ $\mathrm{mm}, \quad \mathrm{h}_{\mathrm{L} 2}=1.0 \mathrm{~mm}, A=20.0 \mathrm{~mm}, w=s=2.0 \mathrm{~mm}$, $\varepsilon_{\mathrm{rL} 1}=\varepsilon_{\mathrm{rU} 1}=1.0, \varepsilon_{\mathrm{rL} 2}=9.35$ )

After testing the method we applied it to the TWFET structure. First we compared the dispersion diagram obtained from our FDTD method with the results previously obtained with the mode matching technique [2]

Fig 6 shows a similar quasi-TEM behavior for the first three modes the structure, moreover showing the existence of a higher order mode, whose cut-off frequency is sufficiently high not to disturb the TWFET behavior (cutoff frequency $=30 \mathrm{GHz}$ ).

Then, as previously stated, we checked the effect of a $T$ gate on the dispersion diagram of the structure. As observed in Fig. 7 the presence of an actual T structure slightly affects the fundamental mode's behaviour that remains quasi-TEM and does not perturb the higher order mode. On the contrary a small increasing of the phase- 
velocity of two of the three fundamental modes is observed, with a small reduction of component performances.

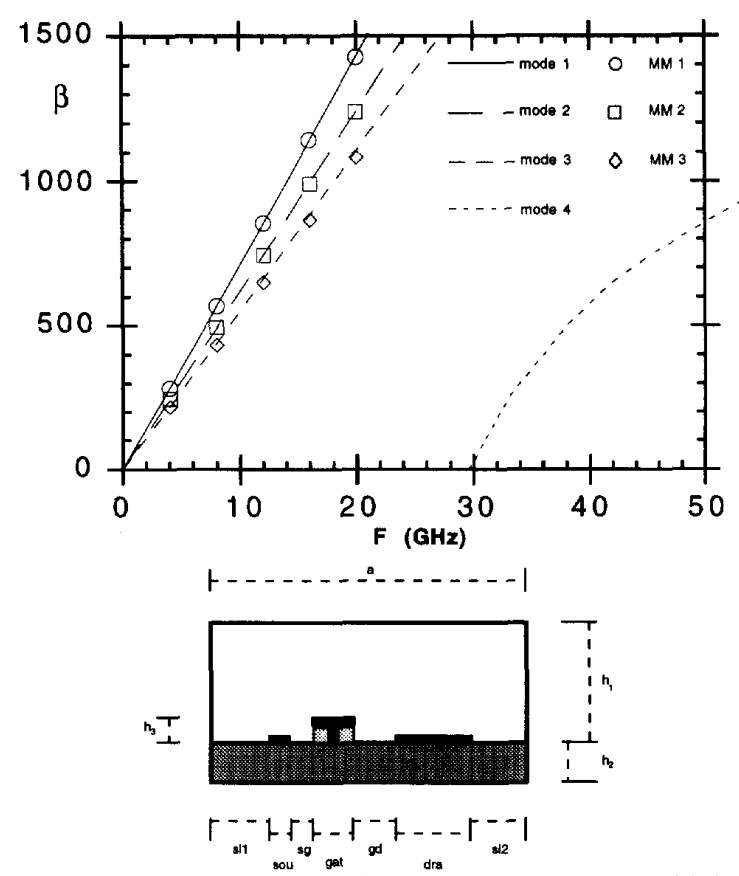

Fig.6 Dispersion curves of quasi-TEM modes and higher order mode in a planar TWFET structure. Comparison with results obtained with mode matching method. ( $s=5 \mu \mathrm{m}$, $\mathrm{g}=30 \mu \mathrm{m}, \mathrm{d}=500 \mu \mathrm{m}, \mathrm{sg}=10 \mu \mathrm{m}, \mathrm{gd}=100 \mu \mathrm{m}$, $\mathrm{sl}_{1}=\mathrm{sl}_{2}=800 \mu \mathrm{m}, \mathrm{h}_{1}=30 \mu \mathrm{m}, \mathrm{h}_{2}=5000 \mu \mathrm{m}, \mathrm{h}_{3}=0$, $\varepsilon_{\mathrm{rGaAs}}=12.9$ )

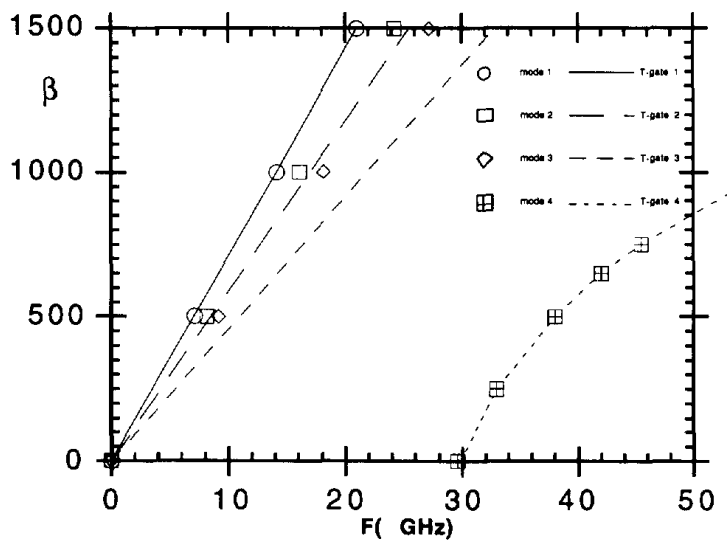

Fig.7 Dispersion curves of quasi-TEM modes and higher order mode in a actual T-gate TWFET structure. Comparison with results shown in fig. $6(\mathrm{~s}=5 \mu \mathrm{m}, \mathrm{g}=30$ $\mu \mathrm{m}, \mathrm{d}=500 \mu \mathrm{m}, \mathrm{sg}=10 \mu \mathrm{m}, \mathrm{gd}=100 \mu \mathrm{m}$, $\mathrm{sl}_{1}=\mathrm{sl}_{2}=800 \mu \mathrm{m}, \mathrm{h}_{1}=30 \mu \mathrm{m}, \mathrm{h}_{2}=5000 \mu \mathrm{m}, \mathrm{h}_{3}=10 \mu \mathrm{m}$, $\varepsilon_{\mathrm{rGaAs}}=12.9, \varepsilon_{\mathrm{rSiO}} 2=3.9$ )

\section{CONCLUSION}

A new 2D-FDTD method has been proposed to analyze the dispersion diagram of planar circuits. The technique has been tested on some cases known from literature and used in the TWFET characterization.

\section{REFERENCES}

[[1] S. D'Agostino, G. D'Inzeo, L. Tudini, "Analytical modeling and design criteria for traveling-wave FET amplifiers" IEEE Transactions on Microwave Theory and Techniques, vol. MTT-40, pp. 202-208, Feb. 1992.

[2] F. Alessandri, G. Baini, G. D'Inzeo, R. Sorrentino, "Propagation characteristics of lossy distribuited GaAs FET structures", IEEE MTT International Microwave Symposium Dygest, Albuquerque (USA), pp 963-966, June 1992

[3] A. Asi, L. Shafai, "Dispersion analysis of anisotropic inhomogeneous waveguides using compact 2D-FDTD" Electronic Letters, vol. 28, no. 15, pp. 1451-1452, Jul. 1992.

[4] S. Xiao, R. Vahldieck, H. Jin, "Full-wave analysis of guided wave structures using a novel 2-D FDTD" IEEE Microwave and Guided Wave Letters, vol. 2, no. 5, pp. 165-167, May 1992.

[5] S. Xiao, R. Vahldieck, "An efficient 2-D FDTD algorithm using real variables" IEEE Microwave and Guided Wave Letters, vol. 3, no. 5, pp. 127-129, May 1993.

[6] A. C. Cangellaris, "Numerical stabilityand numerical dispersion of a compact 2D/FDTD method used for the dispersion analysis of waveguides" IEEE Microwave and Guided Wave Letters, vol. 3, no. 1, pp. 3-5, Jan. 1993.

[7] S. Xiao, R. Vahldieck, "An improved 2D-FDTD algorithm for hybrid mode analysis of quasi-planar transmission lines" 1993 IEEE MTT-S International Microwave Symposium Dygest, pp. 421-424 1993

[8] Sadiku, "Numerical techniques in Electromagnetism", chapter 3, CRC Press, 1992

[9] F. Alessandri, U. Goebel, F. Melai, R. Sorrentino, "Theoretical and experimental characterization of nonsymmetrically shielded coplanar waveguides for millimeter-wave circuits" IEEE Transactions on Microwave Theory and Techniques, vol. MTT-37, pp. 2020-2027, Dec. 1989.

10] R. Lyons, J. P. K. Gilb, C. Balanis, "Enhanced dominant mode operation of a shielded multilayer coplanar waveguide via substrate compensation" IEEE Transactions on Microwave Theory and Techniques, vol. MTT-41, pp. 1564-1567, Sep. 1993.

[11] D. Mirshekar-Syahkal, "Spectral domain method for microwave integrated circuits", Research Studies, 1990

[12] "HFSS-High Frequency Structure Simulator", Hewlett Packard, May 1992 